\title{
A REMARK ON FINELY HARMONIC MAPPINGS
}

\author{
ILPO LAINE
}

B. Fuglede developes in [4] the basic theory of finely harmonic mappings thus generalizing into a finely open domain of definition the concept of a harmonic mapping. This remark presents a small completion of [4]. More precisely, we shall prove some results for covering properties of Bl-mappings (in the fine sense) corresponding to Theorem 3.1.5 and Theorem 3.1.6 in [6], where an attempt to consider finely harmonic mappings was done. After having defined the notion of a fine Bl-mapping (Definition 2) we bring some further details in order to prove the existence of non-trivial fine Bl-mappings (Corollary 7). The results dealing with covering properties begin at Theorem 8 below.

Let $X$ and $X^{\prime}$ be two harmonic spaces in the sense of Constantinescu and Cornea ([1], p. 30) with a countable base and satisfying the domination axiom $D$. We shall apply here mainly the notations introduced in [1], [2], [5] and [6] the exceptions being self-explanatory. Specially we shall denote by $U$ (resp. $U^{\prime}$ ) a finely open set contained in $X$ (resp. $X^{\prime}$ ), unless otherwise explicitly stated.

We first record the following lemma (see for [3], p. 264), the original proof of which carries over into the present axiomatic situation:

Lemma 1. Any two points $x$ and $y$ belonging to a fine domain $U \subseteq X$ can be joined by a (usual) continuum contained in $U$.

Definition 2. A finely harmonic mapping $\varphi: U \rightarrow X^{\prime}$ (in the sense of [4], Theorem 5) is called a fine Bl-mapping into a finely open set $U^{\prime} \supseteqq \varphi(U)$ (and will be called here a fine Bl-mapping $\left.\varphi: U \rightarrow U^{\prime}\right)$, if $p^{\prime} \circ \varphi$ is a fine potential on $\varphi^{-1}\left(V^{\prime}\right)$ for any fine domain $V^{\prime} \subseteq U^{\prime}$ such that $\varphi^{-1}\left(V^{\prime}\right) \neq \emptyset$ and any finite fine potential $p^{\prime}$ on $V^{\prime}$.

Lemma 3. Let $V$ be a finely open set contained in $U$ such that there is a finite fine potential $>0$ on $U$. Then there is a finite fine potential $>0$ on $V$.

Proof. Let $p>0$ be a finite fine potential on $U$ and suppose that $q=0$ is the only finite fine potential on $V$. Hence by [2], Theorem 10.7, any finite fine poten- 
tial on $U$ is finely harmonic on $V$. Let $B \subseteq V$ be a non-empty base. Then $\left(\hat{R}_{p}^{B}\right)_{U}$ is a finite fine potential on $U$ and finely harmonic on $U \backslash B$ by [2], Corollary 11.13, hence finely harmonic on the whole $U$ by the sheaf property see above of finely harmonic functions. Clearly $\left(\hat{R}_{p}^{B}\right)_{U}=0$, a contradiction, since $\left(\hat{R}_{p}^{B}\right)_{U}=p>0$ on $B$ by [2], Lemma 11.10.

Lemma 4. Let $u \geqq 0$ be a finite finely hyperharmonic function on $U$ contained in a $\mathfrak{x}$-set $Y \leqq X$. For each $x \in U$ there is a (finely closed) fine neighbourhood $W \subseteq U$ of $x$ and a finite fine potential $q$ on $U$ such that $u=q$ on $W$.

Proof. Given $x \in U$ let, by Lemma 3, $p$ be a finite fine potential on $U$ such that $u(x)<p(x)$. Let $W$ be a fine neighbourhood of $x$ such that $u \leqq p$ on $W$. Then clearly $\left(\hat{R}_{u}^{W}\right)_{U} \leqq p$, hence $\left(\hat{R}_{u}^{W}\right)_{U}$ is a finite fine potential on $U$.

Using the local extension property ([2], Theorem 9.9) we may assume that $u$ is majorized on $W$ by a locally bounded (usual) potential on $Y$. Further, it is no restriction to assume that $W$ is a base in $Y$, hence $q=\left(R_{u}^{W}\right)_{U}=\left(\hat{R}_{u}^{W}\right)_{U}$ by [2], Lemma 11.5. The lemma follows.

Lemma 5. Let $u \geqq 0$ be a finite finely hyperharmonic function on a finely open set $V$. Let $U \supseteqq V$ be contained in a $\mathfrak{P}$-set $Y \leqq X$. For each point $x \in V$ there is a fine neighbourhood $W \subset V$ of $x$ and two finite fine potentials $p, q$ on $U$ such that $q$ is finely harmonic on $W$ and that $u=p-q$ on $W$.

Proof. By [2], Theorem 9.9, we may take a fine neighbourhood $W \subset V$ of $x$ and two locally bounded potentials $p_{0}, q_{0}$ on $Y$ such that $q_{0}$ is finely harmonic on $W$ and that $u=p_{0}-q_{0}$ on $W$. Let $p_{0}=p_{1}+h_{1}$ (resp. $q_{0}=p_{2}+h_{2}$ ) be the decomposition of $p_{0}$ (resp. $q_{0}$ ) into a finite fine potential and a finely harmonic function on $U$. Hence the decomposition

$$
u=p_{1}+h_{1}-\left(p_{2}+h_{2}\right)
$$

holds on $W$. By the preceding lemma we may assume the existence of two finite fine potentials $q_{1}, q_{2}$ on $U$ such that $h_{1}=q_{1}$ and $h_{2}=q_{2}$ on $W$. Hence

$$
u=p_{1}+q_{1}-\left(p_{2}+q_{2}\right)
$$

on $W$. Clearly $p_{2}+q_{2}$ is finely harmonic on $W$. The assertion follows.

Theorem 6. Let $\varphi: U \rightarrow X^{\prime}$ be a finely harmonic mapping and assume that $U^{\prime} \supseteqq \varphi(U)$ is contained in a $\mathfrak{B}$-set $Y^{\prime} \leqq X^{\prime}$. If $p^{\prime} \circ \varphi$ is a fine potential on $U$ for all finite fine potentials $p^{\prime}$ on $U^{\prime}$, then $\varphi$ is a fine Bl-mapping into $U^{\prime}$.

Proof. Let $V^{\prime}$ be a fine domain $\subseteq U^{\prime}$ such that $\varphi^{-1}\left(V^{\prime}\right) \neq \emptyset$ and let $p^{\prime}$ be a finite fine potential on $V^{\prime}$. Since $p^{\prime} \circ \varphi$ is finite valued, it is finely superharmonic on $\varphi^{-1}\left(V^{\prime}\right)$. Let $h$ be a finely harmonic minorant of $p^{\prime} \circ \varphi$ and denote

$$
\mathfrak{S}^{\prime}=\left\{s^{\prime} \geqq 0 \mid s^{\prime} \text { a fine potential on } V^{\prime} ; s^{\prime} \leqq p^{\prime} ; s^{\prime} \circ \varphi \geqq h\right\} .
$$

It is sufficient to prove that inf $\mathfrak{S}^{\prime}$ is finely harmonic on $V^{\prime}$, since then inf $\mathfrak{S}^{\prime}$ vanishes being dominated by a finite fine potential $p^{\prime}$ ([2], p. 105). 
Let now $x^{\prime} \in V^{\prime}$ be arbitrary. By Lemma 5 there is a finely open fine neighbourhood $W^{\prime}$ of $x^{\prime}$ such that $p^{\prime}$ has a representation $p^{\prime}=p_{1}^{\prime}-p_{2}^{\prime}$ on $\mathrm{cl}_{f} W^{\prime} \subseteq V^{\prime}$, where $p_{1}^{\prime}$ and $p_{2}^{\prime}$ are finite fine potentials on $U^{\prime}$. Let then $T^{\prime}$ be a (fixed) regular finely open set such that $x^{\prime} \in T^{\prime} \subseteq \mathrm{cl}_{f} T^{\prime} \subseteq W^{\prime}$. Let $s^{\prime} \in \Xi^{\prime}$ be arbitrary. By [2], Theorem 10.2, $\left(s^{\prime}\right)^{V^{\prime} \backslash T^{\prime}}$ (defined on $V^{\prime}$ ) is finely harmonic on $T^{\prime}$, $\equiv s^{\prime}$ on $V^{\prime}$ and $=s^{\prime}$ on $V^{\prime} \backslash T^{\prime}$. Therefore $\left(s^{\prime}\right)^{V^{\prime} \backslash T^{\prime}}=s^{\prime}$ holds on $\partial_{f} T^{\prime}$, hence $\left(s^{\prime}\right)^{V^{\prime} \backslash T^{\prime}} \circ \varphi \geqq h$ on $\varphi^{-1}\left(\partial_{f} T^{\prime}\right) \supseteqq \partial_{f} \varphi^{-1}\left(T^{\prime}\right)$. In addition, we have

$$
\left(s^{\prime}\right)^{V^{\prime} \backslash T^{\prime}} \circ \varphi-h \geqq-h \geqq-p^{\prime} \circ \varphi=-p_{1}^{\prime} \circ \varphi+p_{2}^{\prime} \circ \varphi \geqq-p_{1}^{\prime} \circ \varphi
$$

on $\varphi^{-1}\left(T^{\prime}\right)$. Since $\varphi$ is a fine Bl-mapping into $U^{\prime}, p_{1}^{\prime} \circ \varphi$ is a finite fine potential on $U$. By the fine boundary minimum principle ([2], Theorem 10.8) we get $\left(s^{\prime}\right)^{V^{\prime} \backslash T^{\prime}} \circ \varphi \equiv h$ in $\varphi^{-1}\left(T^{\prime}\right)$, hence in $\varphi^{-1}\left(V^{\prime}\right)$. Therefore $\left(s^{\prime}\right)^{V^{\prime} \backslash T^{\prime}} \in \mathfrak{S}^{\prime}$. Since $s^{\prime} \in \mathfrak{S}^{\prime}$ was taken arbitrarily, this implies that inf $\mathfrak{S}^{\prime}$ is finely harmonic on $T^{\prime}$. By the sheaf property of finely harmonic functions, inf $\Xi^{\prime}$ is finely harmonic on the whole $V^{\prime}$. The theorem follows.

Corollary 7. A Bl-mapping $\varphi: U \rightarrow U^{\prime}$ (for the definition, see [5], Definition 2.1.2) defined on an open set $U$ into an open $\$$-set $U^{\prime}$ is a fine Bl-mapping into $U^{\prime}$.

Proof. By [4], p. 116, $\varphi$ is a finely harmonic mapping. Let now $p^{\prime}$ be a finite fine potential on $U^{\prime}$. By [2], Theorem 10.12, $p^{\prime}$ is a semibounded potential on $U^{\prime}$, hence $p^{\prime} \circ \varphi$ is a semibounded potential on $U$ ([6], Theorem 2.2.1), hence a fine potential on $U$. The assertion follows by the preceding theorem.

Remark. The above corollary could have been proved directly exactly like Theorem 6 by using the local extension property ([2], Theorem 9.9) instead of Lemma 5. Based on a discussion with B. Fuglede we have preferred the above more general approach which proves for fine Bl-mappings an analogon to the (well-known) fact, that a harmonic mapping $\varphi: U \rightarrow U^{\prime}$ defined on an open set $U$ into an open P-set $U^{\prime}$ is a Bl-mapping, if $p^{\prime} \circ \varphi$ is a potential on $U$ for all locally bounded potentials $p^{\prime}$ on $U^{\prime}$.

The following theorem is a slight reformulation of a result due to Fuglede ([4], Theorem 9). The proof given by Fuglede carries over, when we only note, that $\varphi: V \rightarrow V^{\prime}$ is a finely open fine Bl-mapping.

Theorem 9. Let $\varphi: U \rightarrow U^{\prime}$ be a finely open fine Bl-mapping. If there is a finite fine potential $>0$ on a fine domain $V^{\prime} \subseteq U^{\prime}$ and if $V$ is the union of a nonempty subfamily of the fine components of $\varphi^{-1}\left(V^{\prime}\right) \neq 0$, then $V^{\prime} \backslash \varphi(V)$ is a polar set.

Let $\mathfrak{G}_{f}(x)$ denote the family of finely open finely connected relatively compact fine neighbourhoods of $x \in U$ (resp. $x \in X^{\prime}$ ). For a finely harmonic mapping $\varphi: U \rightarrow X^{\prime}$ we denote

$$
\bar{n}\left(\varphi, x^{\prime}, A\right)=\min \left(\aleph_{0}, \operatorname{card}\left(A \cap \varphi^{-1}\left(x^{\prime}\right)\right)\right)
$$


for all $A \subseteq U$ and define the local and total multiplicities of $\varphi$ in the fine sense as follows:

$$
\begin{gathered}
n_{f}(\varphi, x)=\inf _{G \in \mathfrak{G}_{f}(x)}\left(\sup _{x^{\prime} \in X^{\prime}} \bar{n}\left(\varphi, x^{\prime}, G\right)\right), x \in U, \\
n_{f}\left(\varphi, x^{\prime}, A\right)=\min \left(\aleph_{0}, \sum_{x \in A \cap \varphi^{-1}\left(x^{\prime}\right)} n_{f}(\varphi, x)\right), A \leqq U
\end{gathered}
$$

(see [6], Section 2.3). The usual local and total multiplicities of $\varphi$ will be defined similarly with respect to the usual neighbourhoods (see [5], Section 1.3).

Lemma 9. Let $\varphi: U \rightarrow U^{\prime}$ be a finely continuous mapping between two finely open sets. If $\varphi^{-1}\left(x^{\prime}\right)=\left\{z_{1}, \ldots, z_{p}\right\}$ is a finite set, then for each $k \leqq p$ there is $G^{\prime} \in \mathfrak{G}_{f}\left(x^{\prime}\right)$ such that some disjoint fine components $V_{1}, \ldots, V_{k}$ of $\varphi^{-1}\left(G^{\prime}\right)$ satisfy $z_{i} \in V_{i}$ for $i=1, \ldots, k$.

Proof. The case $k=1$ being trivial, let us consider the case $k=2$. Let $\left(U_{i}^{\prime}\right)_{i \in N}$ be a sequence of relatively compact (usual) neighbourhoods of $x^{\prime}$ such that $\cap_{i=1}^{\infty} U_{i}^{\prime}=\left\{x^{\prime}\right\}$. We may take a decreasing sequence $\left(G_{i}^{\prime}\right)_{i \in \mathrm{N}}$ of fine neighbourhoods in $\tilde{b}_{f}\left(x^{\prime}\right)$ such that $G_{i}^{\prime} \subseteq U_{i}^{\prime} \cap U^{\prime}$ for each $i \in \mathbf{N}$. Clearly $\bigcap_{i=1}^{\infty} G_{i}^{\prime}=\left\{x^{\prime}\right\}$. Let now, for each $i \in \mathbf{N}, V_{i}$ be the fine component of $\varphi^{-1}\left(G_{i}^{\prime}\right)$ containing $z_{1}$. If the assertion fails for $k=2$, then certainly $z_{2} \in V_{i}$ for all $i \in \mathbf{N}$. We first note that

hence

$$
\bigcap_{i=1}^{\infty} V_{i} \subseteq \bigcap_{i=1}^{\infty} \varphi^{-1}\left(G_{i}^{\prime}\right)=\varphi^{-1}\left(\bigcap_{i=1}^{\infty} G_{i}^{\prime}\right)=\varphi^{-1}\left(x^{\prime}\right)=\left\{z_{1}, \ldots, z_{p}\right\},
$$

$$
\mathrm{cl}\left(\bigcap_{i=1}^{\infty} V_{i}\right) \subseteq\left\{z_{1}, \ldots, z_{p}\right\} .
$$

By Lemma 1 there is a (usual) continuum $C_{i}$ such that $\left\{z_{1}, z_{2}\right\} \subseteq C_{i} \subseteq V_{i}$ for each $i \in \mathbf{N}$. Let now $W_{1}, \ldots, W_{p-1}$ be relatively compact (usual) neighbourhoods of $z_{1}$ such that $z_{2} \notin\left(\mathrm{cl} W_{1}\right) \cup \ldots \cup\left(\mathrm{cl} W_{p-1}\right)$ and that the relative boundaries $\partial W_{1}, \ldots$ $\ldots, \partial W_{p-1}$ are pairwise disjoint. Consider an arbitrary $j \in\{1, \ldots, p-1\}$. Obviously we may take $x_{i} \in C_{i} \cap \partial W_{j}$ for each $i \in \mathbf{N}$. Let $y_{i} \in \partial W_{j}$ be a (usual) cluster point of the sequence $\left(x_{i}\right)_{i \in N}$ and let $T$ be any (usual) neighbourhood of $y_{j}$. Clearly $T$ contains an infinite number of points of the sequence $\left(x_{i}\right)_{i \in N}$, hence $V_{i} \cap T \supseteqq\left\{x_{i}\right\} \neq \emptyset$ for infinitely many $i \in \mathbf{N}$. Since $\left(V_{i}\right)_{i \in N}$ is a decreasing sequence, $T \cap \cap_{i=1}^{\infty} V_{i} \neq \emptyset$, hence we get

$$
y_{j} \in \operatorname{cl}\left(\bigcap_{i=1}^{\infty} V_{i}\right) \subseteq\left\{z_{1}, \ldots, z_{p}\right\} .
$$

Since $j$ was arbitrary, we have $\left\{z_{1}, z_{2}, y_{1}, \ldots, y_{p-1}\right\} \subseteq \varphi^{-1}\left(x^{\prime}\right)$, a contradiction.

The final inductive step of the proof is obvious, see e.g. the proof of Lemma 1.3.4 in [5]. The lemma follows.

We are now ready to state our main results (Theorem 10 and Theorem 11). Their proofs can be omitted, because they are completely similar to the proofs 
of Theorem 3.1.5 and Theorem 3.1.6 in [6]. We must only make some preliminary remarks. Firstly, parallel results to [6], Lemma 3.1.3 and Corollary 3.1.4 hold also in the present situation. Secondly, we observe that for each finely closed non-polar set $E^{\prime} \subseteq U^{\prime}$ there is a point $x^{\prime} \in E^{\prime}$ such that $E^{\prime} \cap G^{\prime}$ is non-polar for all $G^{\prime} \in \mathfrak{G}_{f}\left(x^{\prime}\right)$ by the quasi Lindelöf principle ([6], Lemma 2.1.9). Thirdly, the complement $U^{\prime} \backslash A^{\prime}$ of a polar set $A^{\prime} \subseteq U^{\prime}$ relative to a fine domain $U^{\prime}$ is finely connected. Finally, in the proofs we may use Theorem 8, Lemma 3 and Lemma 9 instead of [6], Theorem 3.1.2, and [5], Lemma 1.3.4, which were used in [6]. One should also note that the proof of [6], Theorem 3.1.6 (applied for Theorem 11 below) actually carries over without making use of minimal neighbourhoods (in the fine sense).

Theorem 10. Let $\varphi: U \rightarrow U^{\prime}$ be a finely open fine Bl-mapping such that

$$
\left(D_{\varphi}^{\prime}\right)_{f}=\left\{x^{\prime} \in U^{\prime} \mid \exists x \in \varphi^{-1}\left(x^{\prime}\right) \text { with } n_{f}(\varphi, x)>1\right\}
$$

is a polar set. If $V^{\prime} \subseteq U^{\prime}$ is a fine domain such that there is a finite fine potential $>0$ on $V^{\prime}$ and if $V$ is the union of a non-empty subfamily of the fine components of $\varphi^{-1}\left(V^{\prime}\right) \neq \emptyset$, then

$$
\left\{x^{\prime} \in V^{\prime} \mid \bar{n}\left(\varphi, x^{\prime}, V\right) \leqq p\right\}
$$

is a polar set for each $p<\bar{k}=\sup _{x^{\prime} \in V^{\prime}} \bar{n}\left(\varphi, x^{\prime}, V\right)$.

Theorem 11. Let $\varphi: U \rightarrow U^{\prime}$ be a finely open fine Bl-mapping such that $\left(D_{\varphi}^{\prime}\right)_{f}$ is a polar set. If $V^{\prime} \subseteq U^{\prime}$ is a fine domain such that there is a finite fine potential $>0$ on $V^{\prime}$ and if $V$ is the union of a non-empty subfamily of fine components of $\varphi^{-1}\left(V^{\prime}\right) \neq \emptyset$, then

and

$$
\bar{k}=\sup _{x^{\prime} \in V^{\prime}} \bar{n}\left(\varphi, x^{\prime}, V\right)=\sup _{x^{\prime} \in V^{\prime}} n_{f}\left(\varphi, x^{\prime}, V\right)=k_{f}
$$

$$
\left\{x^{\prime} \in V^{\prime} \mid n_{f}\left(\varphi, x^{\prime}, V\right) \leqq p\right\}
$$

is a polar set for each $p<\bar{k}$.

The above reasoning can be now applied to get an improvement (under some restrictions to the harmonic spaces) to our earlier results ([5], Theorem 2.2.9, [6], Theorem 3.2.3, [7], Theorem 4.4 and Theorem 5.6) concerning with the covering properties of usual Bl-mappings.

Theorem 12. Let $U$ and $U^{\prime}$ be open sets such that all points of $U^{\prime}$ are polar. If $\varphi: U \rightarrow U^{\prime}$ is a Bl-mapping such that

$$
D_{\varphi}^{\prime}=\left\{x^{\prime} \in U^{\prime} \mid \exists x \in \varphi^{-1}\left(x^{\prime}\right) \text { with } n(\varphi, x)>1\right\}
$$

is a polar set, if $V^{\prime} \subseteq U^{\prime}$ is a $\mathfrak{P}$-domain and if $V$ is the union of a non-empty subfamily of the components of $\varphi^{-1}\left(V^{\prime}\right) \neq \emptyset$, then

$$
\left\{x^{\prime} \in V^{\prime} \mid n\left(\varphi, x^{\prime}, V\right) \leqq p\right\}
$$

is a polar set for each $p<k=\sup _{x^{\prime} \in V^{\prime}} n\left(\varphi, x^{\prime}, V\right)=k_{f}=\bar{k}$. 
Proof. We must only note that $\varphi$ is a finely open finely harmonic mapping, hence the proofs of Theorem 3.1.5 and Theorem 3.1.6 in [6] are again applicable.

Remark. In conclusion we mention that in the situation of Theorem 12 $\left(D_{\varphi}^{\prime}\right)_{f}$ is a polar set if and only if $D_{\varphi}^{\prime}$ is polar. The inclusion $\left(D_{\varphi}^{\prime}\right)_{f} \sqsubseteq D_{\varphi}^{\prime}$ is trivial. If, on the other hand, $\left(D_{\varphi}^{\prime}\right)_{f}$ is a polar set while $D_{\varphi}^{\prime}$ is non-polar, then we may take a $\mathfrak{P}$-domain $V^{\prime} \subseteq U^{\prime}$ such that $V^{\prime} \cap D_{\varphi}^{\prime}$ is a non-polar set. The assumptions of Theorem 10 above are now satisfied for the restricted mapping $\varphi: \varphi^{-1}\left(V^{\prime}\right) \rightarrow V^{\prime}$, hence the conclusion of Theorem 10 holds. The method of the proof of Theorem 3.1.6 in [6] can be once more applied, hence $\bar{k}=k_{f}=k$, therefore we get immediately a contradiction to Theorem 10 .

Acknowledgement. This work has been done while the author was visiting the Department of Mathematics, University of Erlangen-Nürnberg, supported by the Alexander von Humboldt Foundation.

\section{References}

[1] Constantinescu, C., and A. Cornea: Potential theory of harmonic spaces. - Springer-Verlag, Berlin-Heidelberg-New York, 1972.

[2] Fuglede, B.: Finely harmonic functions. - Lecture Notes in Mathematics 289, SpringerVerlag, Berlin-Heidelberg-New York, 1972.

[3] Fuglede, B.: Asymptotic paths for subharmonic functions. - Math. Ann. 213, 1975, $261-274$.

[4] Fuglede, B.: Finely harmonic mappings and finely holomorphic functions. - Ann. Acad. Sci. Fenn. Ser. A I 2, 1976, 113-127.

[5] LaINE, I.: Covering properties of harmonic Bl-mappings I. - Rev. Roumaine Math. Pures Appl. 19, 1974, 21-34.

[6] LAINE, I.: Covering properties of harmonic Bl-mappings II. - Ann. Acad. Sci. Fenn. Ser. A I $570,1974,1-14$.

[7] LAINE, I.: Covering properties of harmonic Bl-mappings III. - Ibid. 1, 1975, 309-325.

University of Joensuu

Department of Mathematics and Physics

SF-80101 Joensuu 10

Finland

Received 14 April 1976 